\title{
The investigation on the dark sector at the PADME experiment
}

\author{
Paola Gianotti*t \\ Laboratori Nazionali di Frascati of INFN \\ via E. Fermi 40 \\ 00044 Frascati (Italy) \\ E-mail: paola.gianotti@lnf.infn.it
}

The PADME experiment, by using the positron beam of the INFN Frascati laboratory, looks for signals of a dark photon, $A^{\prime}$. By knowing the beam energy, and measuring precisely the fourmomentum of the ordinary recoil photon, a signal is searched in the missing mass of the process $e^{+} e^{-} \rightarrow A^{\prime} \gamma$. The precise determination of this quantity, and the capability to reject background events, are the key points for the success of the experiment. In this paper, a general description of the physics case and of the detector are given.

ICHEP 2018, XXXIX International Conference on High Energy Physics

4-11 July 2018

Seoul, Korea

\footnotetext{
${ }^{*}$ Speaker.

${ }^{\dagger}$ G. Chiodini, F. Gotand, F. Oliva (INFN Lecce), A.P. Caricato, M. Martino, S.Spagnolo (INFN Lecce e Dip. di Matematica e Fisica, Università del Salento), P. Albicocco, F. Bossi, B. Buonomo, R. De Sangro, D. Domenici, G. Finocchiaro, L.G. Foggetta, A. Ghigo, P. Gianotti, G. Piperno, I. Sarra, B. Sciascia, T. Spadaro, E. Spiriti, E. Vilucchi (INFN Laboratori Nazionali di Frascati), L. Tsankov (University of Sofia "St. Kl. Ohridski"), G. Georgiev, V. Kozhuharov (University of Sofia "St. Kl. Ohridski" and INFN Laboratori Nazionali di Frascati), F. Ferrarotto, E. Leonardi, F. Safai Tehrani, P. Valente (INFN Roma1), S. Fiore (INFN Roma1 e ENEA), G.C. Organtini, M. Raggi (INFN Roma1 e Dip. di Fisica, "Sapienza" Università di Roma), B. Liberti, C. Taruggi (INFN Laboratori Nazionali di Frascati e Dip. di Fisica, Università di Roma “Tor Vergata”)
} 


\section{Introduction}

Except for gravitational effects, very little is known about the constituents and interactions of Dark Matter (DM). This is why an intense activity is carried out by many experiments to find signals of weakly interacting massive particles, axions, sterile neutrinos and all other possible DM candidates. Despite the enormous experimental effort, no evidences have been yet found and at present, very little is known about DM nature. Therefore, any new attempt to shed light to the field should be conducted in the more unbiased way spanning a wide range of targets and methodologies.

A simple theoretical well motivated approach to the DM problem postulates the existence of a massive Dark Photon (DP) $A^{\prime}$, related to a new Abelian gauge symmetry $U(1)^{\prime}$ interacting with ordinary matter only through kinetic mixing $\frac{\varepsilon}{2} F^{\mu v} F_{\mu \nu}^{\prime}$ with the SM photon [1]. The smallness of the effective interaction strength $\varepsilon^{2}\left(10^{-8} \leq \varepsilon^{2} \leq 10^{-4}\right)$ would explain why so far, the DP escaped detection. Due to the coupling with the standard model photon, the DP can be produced in electromagnetic processes: electron Bremsstrahlung, $e^{+} e^{-}$annihilation, mesons decays with photons in the final state. Regarding the decay mode of the DP, two possible scenarios named "visible" and "invisible" are foreseen, depending on its mass and on the nature of the particles of the dark sector. If the DP is the lightest of the dark states, it would experience decays into Standard Model leptons or hadrons; on the other hand, if in the dark sector exist lighter particles than the $A^{\prime}$, it would decay through them and it will be undetectable. The investigation techniques for the two scenarios are complementary. In case of visible decays a narrow resonance might be observed in the invariant mass final states spectrum (leptonic or hadronic), while in the invisible case, the DP could manifest itself through missing mass or missing energy. A detailed review of the DP physics and experimental bounds scan be found in ref. [2].

\section{The PADME experiment}

The Positron Annihilation into Dark Matter Experiment (PADME) [3] aims to produce a DP in the process:

$$
e^{+} e^{-} \rightarrow A^{\prime} \gamma
$$

making no assumption on its decays. To measure such a reaction it is used the high intensity, 550 $\mathrm{MeV}$ positron beam of the LINAC of Frascati National Laboratory (LNF) of INFN. By knowing precisely the kinematics of the initial state and measuring the 4-momentum of the ordinary photon, an $A^{\prime}$ signal would manifest as a peak in the missing mass spectrum of the reaction (2.1).

This experimental approach relies on the capability of rejecting efficiently background that can filter within the event selection constraints: $e^{+} e^{-} \rightarrow 2 \gamma, 3 \gamma, e^{+} N \rightarrow e^{+} N \gamma, e^{+} e^{-} \rightarrow e^{+} e^{-} \gamma$. An overview of the PADME layout is show in Fig. 1. It consists in a combination of different elements optimized to perform the measurement described allowing an efficient background rejection:

- a $100 \mu \mathrm{m}$ thin diamond active target where the positrons annihilation takes place, capable to measure the average position and the intensity of the beam, by means of orthogonal, graphitic readout strips created on both faces; 
- two pairs of ultra-thin silicon pixel MIMOSA-28 detectors, that can be inserted in the interaction region, in place of the target, to better monitor beam characteristics in terms of position and divergence;

- a finely segmented, high efficiency and resolution electromagnetic calorimeter system to measure and/or veto final state photons. It consists of two elements: the main ECAL, a cylindrical array of 616 BGO crystals having a central squared hole to allow Bremsstrahlung photons passing by, and the faster Small Angle Calorimeter (SAC) to veto such photons. The SAC is a squared matrix of $25 \mathrm{PbF}_{2}$ Cherenkov crystals placed behind the ECAL hole;

- a dipole magnet providing a field of $0.5 T$ to deflect the primary positrons out of the spectrometer and the calorimeters and allowing charged particles momentum analysis;

- a vacuum chamber, to minimize the unwanted interactions of primary and secondary particles;

- three charged particle detectors revealing the positrons that have radiated a high energy photon in the target (PVeto), the electrons coming from the beam target interaction or from particles which decay to final states with electrons (EVeto), and the positrons which radiated relatively small energy in the target (HEPVeto). The three stations consist of plastic scintillator strips readout by SiPMs. They are placed on the left (PVeto), right (EVeto) and back (HEPVeto) sides of the vacuum chamber, respectively. They allow momentum resolution at the level of 5\% for particles in the energy range 50-400 $\mathrm{MeV}$;

- a further silicon pixel detector based on TimePix 3 chip, is placed on the exit path of the positron beam, to monitor the not interacting particles.

A DP event candidate requires a single photon detected by the ECAL, no clusters in the SAC, no signals in the veto counters.
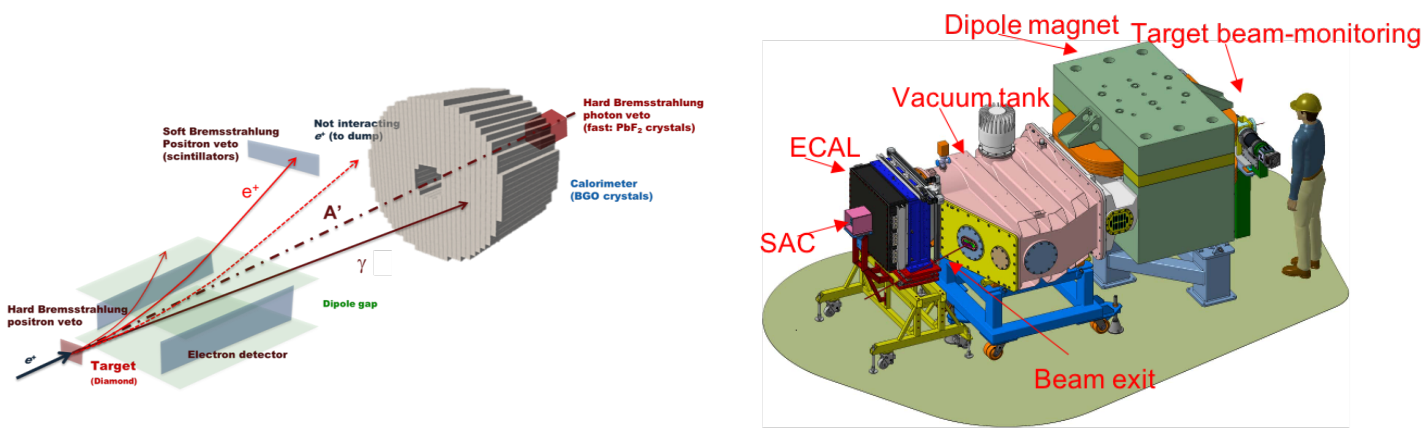

Figure 1: Left. Scheme of the PADME experiment showing the main components and their function. Right. 3D view of the PADME setup. The main components are highlighted (see text for more details. 


\section{The PADME scientific program}

The main goal of the PADME experiment is to collect $10^{13}$ positrons on target in a period of 1-2 years to place a limit on the coupling of the DP down to $3 \times 10^{-4}$ for $A^{\prime}$ masses up to $\sim 24$ $\mathrm{MeV} / \mathrm{c}^{2}$. The running time needed is strongly related to the beam characteristics and the detector performance. At present beam pulses up to 20.000 positron in a time window of about $200 \mathrm{~ns}$ can be sent to the experiment and the commissioning of the detector is ongoing. Figure 2 shows a screen shot of the PADME monitoring system from which it can be seen that all detector components are up and running. The first scientific goal of the PADME experiment is a precise evaluation of all background components. This is, first of all, a need, in order to provide a solid outcome in the field of DM. Furthermore, precise measurements of Bremsstrahlung cross-sections for positrons in the $100 \mathrm{MeV}$ energy range are highly desirable to improve the reliability of Monte Carlo simulation packages. Other channels where PADME will provide valuable input for the tuning of various event generators are the multi-photon annihilation processes.

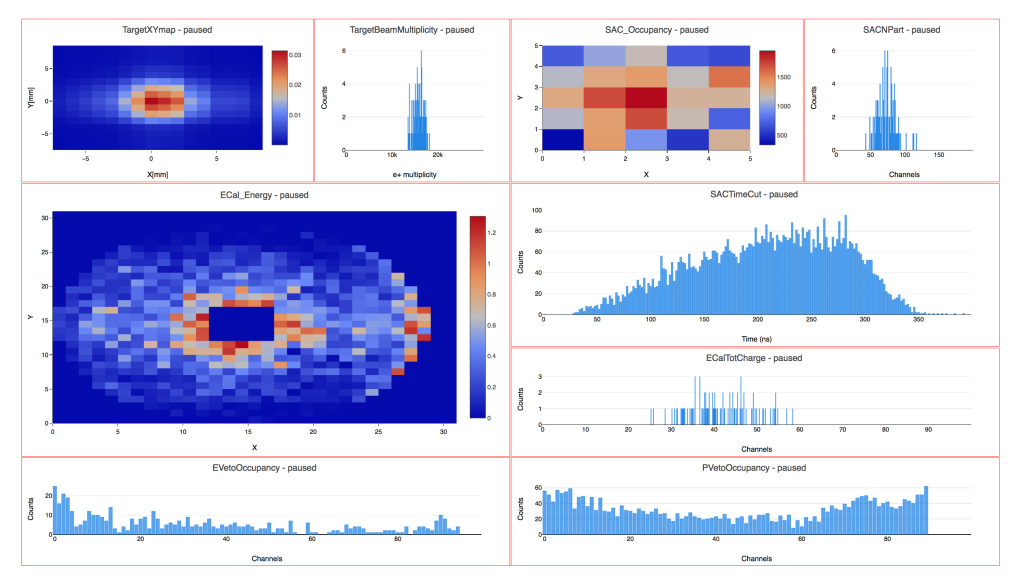

Figure 2: A screenshot of the PADME monitoring system showing the occupancy of different sub-detectors during one of the commissioning runs.

Exploiting the capability of the experiment to detect photon and charged particles, the PADME experiment will be able to investigate also other type of dark sectors mediators such as Axion-LikeParticles, proto-phobic $\mathrm{X}$ boson, and Dark Higgs.

\section{Acknowledgement}

This work is partly supported by the Italian Ministry of Foreign Affairs and International Cooperation (MAECI) under the grant PRG00226 (CUP I86D16000060005).

\section{References}

[1] B. Holdom, Phys. Lett. B 166, 196 (1986).

[2] M. Battaglieri et al., arXiv:1707.04591.

[3] M. Raggi and V. Kozhuharov, Adv. High Energy Phys. 2014, 959802 (2014). 